\title{
RIEMANNIAN MANIFOLDS STRUCTURED BY A LOCAL CONFORMAL SECTION
}

\author{
By
}

Filip DeFEVER and Radu RosCA

\begin{abstract}
Geometrical and structural properties are proved for manifolds which are structured by the presence of a local conformal section.
\end{abstract}

\section{Introduction}

Let $(M, g)$ be an $n$-dimensional Riemannian manifold and let $\mathcal{O}=$ vect $\left\{e_{i} \mid i=1, \ldots n\right\}$ be a local field of orthonormal frames over $M$ and let $\mathcal{G}^{*}=\operatorname{covect}\left\{\omega^{i} \mid i=1, \ldots n\right\}$ be its associated coframe. Let $\alpha=\sum_{i=1}^{n} t_{i} \omega^{i}$ be a globally defined 1 -form and let $\mathscr{T}=\alpha^{\#}$ be its dual vector field. If the connection forms $\theta$ associated with $\mathcal{O}$ satisfy

$$
\left\langle e_{i} \wedge e_{j}, \mathscr{T}\right\rangle=\theta_{j}^{i}
$$

we say that $M$ is structured by a local conformal section $\mathscr{T}$.

In the present paper, we prove that in this case $\mathscr{T}$ is a concurrent vector field [2] which satisfies

$$
\nabla_{Z} \mathscr{T}=\rho Z, \quad Z \in \Xi(M), \rho \in C^{\infty}(M)
$$

In consequence of this fact, $\mathscr{T}$ is both a conformal vector field (with $\rho$ as conformal factor) and an exterior concurrent vector field [12]. Moreover, in Section 3 the following properties are also proved:

(i) the dual form, the connection forms, and the curvature forms associated with $\mathcal{O}$ are $d^{-x}$-exact, $d^{-2 x}$-exact, and $d^{-4 \rho x}$-exact, respectively;

(ii) $\mathscr{T}$ commutes with the dual vectors and the connection forms $\theta$;

(iii) the divergences of $e_{i}$ constitute an $m$-dimensional eigenspace of $\Delta$, corresponding to the eigenvalue $-((n+1) / 2) \rho$; 
(iv) the scalar curvature $S$ of $M$ is expressed by

$$
S=-\frac{n^{2}+n-2}{2} \rho
$$

(v) if $\mathscr{U}$ is any parallel vector field, then through Weitzenbock's formula [10] one finds that $g(\mathscr{T}, \mathscr{U})$ is an eigenfunction of $\Delta$.

Next, in Section 4 we study some properties of the Lie algebra of infinitesimal transformations induced by $\mathscr{T}$ and prove:

(i) $\mathscr{T}$ defines an infinitesimal conformal transformation of $S$ and of the function $g(\mathscr{T}, Z)$, for $Z \in \Xi(M)$, which means that

$$
\mathscr{L}_{\mathscr{T}} S=\rho S, \quad \mathscr{L}_{\mathscr{T}} g(\mathscr{T}, Z)=\frac{2 n-3}{n-1} \rho g(\mathscr{T}, Z)
$$

(ii) we denote by $V, \mu, \psi$, and $\boldsymbol{L}$, the canonical vector field, the Liouville 1form [4], the canonical symplectic form on $T M$, and the operator of Yano and Ishihara, respectively; then $\psi$ is a Finslerian form [4] which is invariant by $\mathscr{T}$;

(iii) the complete lift $\Omega^{C}$ of the symplectic form $\Omega$ of $M$ is also conformally symplectic on $T M$;

(iv) the complete lift $\alpha^{C}$ of $\alpha=\mathscr{T}^{b}$ is also an exact form.

\section{Preliminaries}

Let $(M, g)$ be an $n$-dimensional Riemannian manifold and let $\nabla$ be the covariant differential operator defined by the metric tensor. We assume in the sequel that $M$ is oriented and that the connection $\nabla$ is symmetric.

Let $\Gamma T M=\Xi(M)$ be the set of sections of the tangent bundle $T M$, and

$$
b: T M \stackrel{b}{\rightarrow} T^{*} M \quad \text { and } \#: T M \stackrel{\#}{\longleftarrow} T^{*} M
$$

the classical isomorphisms defined by the metric tensor $g$ (i.e. ${ }^{b}$ is the index lowering operator, and \# is the index raising operator).

Following [10], we denote by

$$
A^{q}(M, T M)=\Gamma \operatorname{Hom}\left(\Lambda^{q} T M, T M\right),
$$

the set of vector valued $q$-forms $(q<\operatorname{dim} M)$, and we write for the covariant derivative operator with respect to $\nabla$

$$
d^{\nabla}: A^{q}(M, T M) \rightarrow A^{q+1}(M, T M)
$$

It should be noticed that in general $d^{\nabla^{2}}=d^{\nabla} \circ d^{\nabla} \neq 0$, unlike $d^{2}=d \circ d=0$. 
Furthermore, we denote by $d p \in A^{1}(M, T M)$ the canonical vector valued 1form of $M$, which is also called the soldering form of $M$ [3]; since $\nabla$ is assumed to be symmetric, we recall that the identity $d^{\nabla}(d p)=0$ is valid.

The operator

$$
d^{\omega}=d+e(\omega)
$$

acting on $\Lambda M$ is called the cohomology operator [5]. In (1), e(w) means the exterior product by the closed 1 -form $\omega$, i.e.

$$
d^{\omega} u=d u+\omega \wedge u
$$

with $u \in \Lambda M$. Clearly one has the identity

$$
d^{\omega} \circ d^{\omega}=0 .
$$

A form $u \in \Lambda M$ such that

$$
d^{(\omega} u=0
$$

is said to be $d^{\omega}$-closed, and $\omega$ is called the cohomology form.

A vector field $X$ which satisfies

$$
d^{\nabla}(\nabla X)=\nabla^{2} X=\pi \wedge d p \in A^{2}(M, T M), \quad \pi \in \Lambda^{1} M,
$$

is defined to be an exterior concurrent vector field [12]. The 1 -form $\pi$ in (2) is called the concurrence form and is defined by

$$
\pi=\lambda X^{b}
$$

where $\lambda \in C^{\propto}(M)$ is a nonzero conformal scalar associated with $X$. If $\mathscr{T}$ is any conformal vector field on $M$, which means that

$$
\mathscr{L}_{\mathscr{T}} g=\rho g \Leftrightarrow\left\langle\nabla_{Z} \mathscr{T}, Z^{\prime}\right\rangle+\left\langle\nabla_{Z^{\prime}} \mathscr{T}, Z\right\rangle=\rho\left\langle Z, Z^{\prime}\right\rangle,
$$

then it follows that

$$
\rho=\frac{2}{n} \operatorname{div} \mathscr{T}
$$

Therefore, in application of Orsted's lemma [1] one can write

$$
\mathscr{L}_{\mathscr{T}} Z^{b}=\rho Z^{b}+[\mathscr{T}, Z]^{p},
$$

where $[$,$] stands for the Lie bracket. If S$ is the scalar curvature of $M$, then Yano's formula [15] reads

$$
\mathscr{L}_{\mathscr{T}} S=(n-1) \Delta \rho-S \rho
$$


Let

$$
\left(\operatorname{Hess}_{\nabla} \rho\right)\left(Z, Z^{\prime}\right)=g\left(Z, H_{\rho} Z^{\prime}\right)
$$

where

$$
H_{\rho} Z^{\prime}=\nabla_{Z^{\prime}} \operatorname{grad} \rho
$$

then

$$
2 \mathscr{L}_{\mathscr{T}} \mathscr{R}\left(Z, Z^{\prime}\right)=(\Delta \rho) g\left(Z, Z^{\prime}\right)-(n-2)\left(\operatorname{Hess}_{\nabla} \rho\right)\left(Z, Z^{\prime}\right) .
$$

In Section 5 we will rely on the following concepts concerning the tangent bundle manifold $T M$ having as basis manifold $M$. Denote by $V\left(V^{i}\right)(i=1, \ldots n)$ the Liouville vector field (or the canonical vector field on $T M$ [6]). Accordingly, one may consider the sets

$$
\mathscr{B}=\left\{e_{i}, \frac{\partial}{\partial V^{i}} \mid i=1, \ldots n\right\}, \quad \text { and } \quad \mathscr{B}^{*}=\left\{\omega^{i}, d V^{i} \mid i=1, \ldots n\right\}
$$

as an adapted vectorial basis, and an adapted cobasis in $T M$, respectively.

For application in the sequel, we remind that the vertical differential operator $d_{V}$ is an antiderivation of degree 1 on $\Lambda(T M)$, and is defined by [4]

$$
d_{V}(f)=\sum_{i=1}^{n} \frac{\partial f}{\partial V^{i}} \omega^{i}, \quad d_{V}\left(\omega^{i}\right)=0, \quad d_{V}\left(d V^{i}\right)=0
$$

the vertical operator $i_{V}$, which is a derivation of degree 0 on $\Lambda(T M)$, is defined by $[4]$

$$
i_{V}(f)=0, \quad i_{V}\left(\omega^{i}\right)=0, \quad i_{V}\left(d V^{i}\right)=\omega^{i}
$$

Moreover, both operators $d_{V}$ and $i_{V}$ satisfy the following relation

$$
\left[d_{V}, i_{V}\right]=d_{V}
$$

Next, with $V$ denoting the Liouville vector field $V$, which may be expressed as [6]

$$
V=\sum_{i=1}^{n} V^{i} \frac{\partial}{\partial V^{i}}
$$

then, by definition, any 1 -form $u$ such that

$$
\mathscr{L}_{v} u=\gamma u
$$

is said to be homogeneous of degree $\gamma$. 
The vertical lift $Z^{V}$ [16] of any vector field $Z$ on $M$ with components $Z^{i}(i=1, \ldots n)$ is expressed by

$$
Z^{V}=\sum_{i=1}^{n} Z^{i} \frac{\partial}{\partial V^{i}}=:\left(\begin{array}{c}
0 \\
Z^{i}
\end{array}\right)
$$

and the complete lift $Z^{C}$ of $Z\left(Z^{i}\right)(i=1, \ldots n)$ is given by

$$
Z^{C}=\sum_{i=1}^{n}\left(Z^{i} e_{i}+\partial Z^{i} \frac{\partial}{\partial V^{i}}\right)=:\left(\begin{array}{c}
Z^{i} \\
\partial Z^{i}
\end{array}\right),
$$

where $\partial Z^{i}=\sum_{\kappa=1}^{n} V^{\kappa} \partial_{\kappa} Z^{i}$, with $\partial_{\kappa}$ the pfaffian derivative.

Finally, the complete lift $\beta^{C}$ of a 1 -form $\beta=\sum_{i=1}^{n} \beta_{i} \omega^{i}$ is defined by

$$
\beta^{C}=\sum_{i=1}^{n}\left(\partial \beta^{i} \omega^{i}+\beta^{i} d V^{i}\right)=:\left(\partial \beta^{i}, \beta^{i}\right) .
$$

\section{Manifolds with a Local Conformal Section}

Considering an $n$-dimensional manifold $(M, g)$, then in terms of the local field of adapted vectorial frames $\mathcal{O}=\operatorname{vect}\left\{e_{i} \mid i=1, \ldots n\right\}$ and its associated coframe $\mathcal{O}^{*}=\operatorname{covect}\left\{\omega^{i} \mid i=1, \ldots n\right\}$, the soldering form $d p$ can be expressed as

$$
d p=\sum_{i=1}^{n} \omega^{i} \otimes e_{i}
$$

and we recall that $E$. Cartan's structure equations can be written as

$$
\begin{aligned}
& \nabla e_{A}=\sum_{B=1}^{n} \theta_{A}^{B} \otimes e_{B}, \\
& d \omega^{A}=-\sum_{B=1}^{n} \theta_{B}^{A} \wedge \omega^{B}, \\
& d \theta_{B}^{A}=-\sum_{C=1}^{n} \theta_{B}^{C} \wedge \theta_{C}^{A}+\Theta_{B}^{A} .
\end{aligned}
$$

In the above equations $\theta$ (respectively $\Theta$ ) are the local connection forms in the tangent bundle $T M$ (respectively the curvature 2-forms on $M$ ).

Let

$$
\alpha=\sum_{i=1}^{n} t_{i} \omega^{i}
$$


be a globally defined 1 -form on $M$ and let $\mathscr{T}=\alpha^{\#}$ be its dual vector field. If the connection forms satisfy

$$
\left\langle e_{i} \wedge e_{j}, \mathscr{T}\right\rangle=\theta_{i}^{j}
$$

then one says that $M$ is structured by a local conformal section $\mathscr{T}$. From (23) and (24) one gets that

$$
\theta_{i}^{j}=t_{i} \omega^{j}-t_{j} \omega^{i}
$$

This implies that

$$
\theta_{i}^{j}(\mathscr{T})=0
$$

which shows that the forms $\theta$ are integral relations of invariance [8]. Now, in consequence of (24), and making use of (23), one finds that

$$
d \omega^{i}=\alpha \wedge \omega^{i} \Rightarrow d \alpha=0 .
$$

Hence, in terms of $d^{\omega}$-cohomology, and in view of (3), one may write that

$$
d^{-\alpha} \omega^{i}=0
$$

i.e. all covectors of $\mathcal{O}^{*}$ are $d^{-\alpha}$-closed.

Since $\mathscr{T}=\sum_{i=1}^{n} t_{i} e_{i}$, and taking into account (20) and (25), it follows that

$$
\nabla e_{i}=t_{i} d p-\omega^{i} \otimes \mathscr{T}
$$

Recalling now that at each point $p \in M, \operatorname{div} Z=\operatorname{Tr}(\nabla Z)=\sum_{i=1}^{n} \omega^{i}\left(\nabla_{e_{t}} Z\right)$, one derives from (29) that

$$
\operatorname{div} e_{i}=(n-1) t_{i}
$$

which provides a geometrical interpretation for the components of $\mathscr{T}$.

On the other hand, on behalf of (27) one gets

$$
d t_{i}=t_{i} \alpha+a \omega^{i}, \quad a \in C^{\infty}(M)
$$

and by exterior differentiation is can be seen that the scalar function $a$ must in fact be a constant. Setting $2 t=\|\mathscr{T}\|^{2}$ for notational brevity, one finds by (31) and (32) that

$$
d t=(2 t+a) \alpha
$$

which shows that $\alpha$ is an exact form. If we put

$$
\rho=2(2 t+a) \in C^{\infty}(M),
$$


one derives that

$$
d \rho=2 \rho \alpha
$$

and

$$
\mathscr{L}_{\mathscr{T}} \omega^{i}=\frac{\rho}{2} \omega^{i}
$$

The above equation expresses that the vector field $\mathscr{T}$ defines an infinitesimal conformal transformation of all covectors of $\mathcal{O}^{*}$; according to a well know definition [8], we say that $\mathscr{T}$ defines a local conformal section of the manifold $M$.

Next, with the help of (29) and (31), we get

$$
\nabla \mathscr{T}=\frac{\rho}{2} d p
$$

which shows that $\mathscr{T}$ is a concurrent vector field [2]. In turn, this implies the following two properties for $\mathscr{T}$ :

(a) $\mathscr{T}$ is a conformal vector field on $M$, i.e.

$$
\mathscr{L}_{\text {可 }} g=\rho g
$$

and

$$
\operatorname{div} \mathscr{T}=\frac{n}{2} \rho
$$

(b) $\mathscr{T}$ is an exterior concurrent vector field [12], which by (34) satisfies

$$
\nabla^{2} \mathscr{T}=\rho \alpha \wedge d p=\rho \mathscr{T}^{b} \wedge d p
$$

Further, invoking (25) and (31) yields

$$
d \theta_{j}^{i}=2 \alpha \wedge \theta_{j}^{i}+2 a \omega^{i} \wedge \omega^{j},
$$

and making use of (24), one finds that the curvature forms $\Theta$ of $M$ can be expressed by

$$
\Theta_{j}^{i}=\alpha \wedge \theta_{j}^{i}+\left(\frac{\rho}{2}+a\right) \omega^{i} \wedge \omega^{j}
$$

In consequence of $(41)$, one finds that the components $\mathscr{R}_{i j}$ of the Ricci tensor $\mathscr{R}$ are

$$
\left\{\begin{array}{l}
\mathscr{R}_{i i}=-(n-2)\left(t_{i}\right)^{2}-n\left(\frac{\rho}{2}-a\right), \\
\mathscr{R}_{i j}=-(n-2) t_{i} t_{j} .
\end{array}\right.
$$


Now it can be observed that for (42) to be consistent with (39), the constant $a$ must vanish. Accordingly, (40) yields

$$
d \theta_{j}^{i}=2 \alpha \wedge \theta_{j}^{i}
$$

and also

$$
\Theta_{j}^{i}=\alpha \wedge \theta_{j}^{i}+\frac{\rho}{2} \omega^{i} \wedge \omega^{j}
$$

Next, taking the exterior differential of (44), one finds by (34) that

$$
d \Theta_{j}^{i}=4 \rho \alpha \wedge \Theta_{j}^{i} .
$$

In terms of cohomology, the above formulas can be interpreted as follows: on the considered manifold, the dual forms, the connection forms, and the curvature forms are $d^{-\alpha}$-exact, $d^{-2 \alpha}$-exact, and $d^{-4 p \alpha}$-exact, respectively.

If we write now $S$ for the scalar curvature of $(M, g)$, then, in consequence of (45) and $a=0$, one gets that

$$
S=-\frac{n^{2}+n-2}{2} \rho
$$

which since $\rho=2 g(\mathscr{T}, \mathscr{T})$ shows that $S$ is always negative. Next, we define

$$
E_{i j}=t_{i} e_{j}-t_{j} e_{i}
$$

for the dual vectors of $\theta_{i}^{j}$. Taking the covariant differential of $E_{i j}$, one finds by (29)

$$
d E_{i j}=\alpha \otimes E_{i j}-\theta_{i}^{j} \otimes \mathscr{T}
$$

and on behalf of (31), one may write

$$
\left[\mathscr{T}, E_{i j}\right]=0 .
$$

Hence, the conformal section $\mathscr{T}$ commutes with all the dual vectors of the connection forms on $M$. Now, by reference to Orsted's lemma [1], it follows in virtue of (49) that

$$
\mathscr{L}_{\mathscr{T}} \theta_{i}^{j}=\rho \theta_{i}^{j}
$$

and by (44) also that

$$
\mathscr{L}_{\mathscr{T}} \Theta_{i}^{j}=2 \rho \Theta_{i}^{j}
$$

The above equations now express that the vector field $\mathscr{T}$ defines an infinitesimal 
conformal transformation [7], not only of the dual forms of $\mathcal{O}^{*}$, but also of the connection and the curvature forms.

Further, since $\delta \alpha=-\operatorname{div} \mathscr{T}$ (where $\delta$ denotes the codifferential operator), then, by (37) and (34), one calculates that

$$
\Delta \alpha=-n \rho \alpha .
$$

This shows that $\alpha$ is an eigenform of the Laplacian with $-n \rho$ as associated eigenvalue. As $\rho$ is always positive, it follows from the nature of the spectrum of the Laplacian operator that a manifold structured by a local conformal section cannot be compact. With the general formula $\Delta v=-\operatorname{div} \operatorname{grad} v$ and using (34), one gets

$$
\Delta t_{i}=-\frac{n+1}{2} \rho t_{i}
$$

which by (30) turns into

$$
\Delta \operatorname{div} e_{i}=-\frac{n+1}{2} \rho \operatorname{div} e_{i}
$$

The above equation expresses that the divergencies of the vector basis $\mathcal{O}$ on $M$ form an $n$-dimensional space $E^{n}(M)$, which is an eigenspace of $\Delta$ corresponding to the eigenvalue $-((n+1) / 2) \rho$. Similarly, one finds by $d t=(2 t+a) \alpha($ see $(32))$ and (31) that

$$
\operatorname{tr} \nabla^{2} \mathscr{T}=-\frac{n-2}{4} \rho \mathscr{T}
$$

and

$$
\|\nabla \mathscr{T}\|^{2}=\frac{n \rho^{2}}{2}
$$

It can be checked that the above equations, in combination with (52), are indeed consistent with Bochner's theorem [10]

$$
2\left\langle\operatorname{tr} \nabla^{2} Z, Z\right\rangle+2\|\nabla Z\|^{2}+\Delta\|Z\|^{2}=0 .
$$

Summarizing, we can formulate the following

THEOREM 3.1. Let $(M, g)$ be an $n$-dimensional Riemannian manifold structured by a local conformal section $\mathscr{T}$ and let $\alpha=\mathscr{T}^{b}$ be the dual form of $\mathscr{T}$. If $\mathcal{O}$ is a local field of orthonormal frames over $M$, then the dual forms, the connection 
forms, and the curvature forms are $d^{-\alpha}$-exact, $d^{-2 \alpha}$-exact, and $d^{-4 p \alpha}$-exact, respectively. Furthermore:

(i) $\mathscr{T}$ commutes with the dual vectors of the connection forms $\theta$ on $M$;

(ii) the divergences of the vector basis on $M$ constitute an eigenspace of $\Delta$ which corresponds to the eigenvalue $-((n+1) / 2) \rho$;

(iii) the scalar curvature $S$ of $M$ is negative and is given by $S=$ $-\left(\left(n^{2}+n-2\right) / 2\right) \rho$

(iv) $\alpha$ is an eigenform of $\Delta$ and the manifold $M$ under consideration can not be compact.

\section{The Lie Algebra of Infinitesimal Transformations}

In this section, we discuss some properties of the Lie algebra of infinitesimal transformations generated by the conformal field $\mathscr{T}$. First, by (36), one may write

$$
\operatorname{grad} \rho=2 \rho \mathscr{T} \Rightarrow\|\operatorname{grad} \rho\|^{2}=2 \rho^{3} .
$$

Therefore,

$$
\operatorname{div}(\operatorname{grad} \rho)=(n+2) \rho^{2} .
$$

The above equations show that $\|\operatorname{grad} \rho\|^{2}$ and $\operatorname{div}(\operatorname{grad} \rho)$ can be expressed as functions of $\rho$. Thus, on behalf of a well known definition [14], it follows that the conformal scalar $\rho$ is an isoparametric function.

Now, by reference to Yano's formula (9) one gets

$$
\mathscr{L}_{\mathscr{T}} S=-\frac{n^{2}+n-2}{2} \rho^{2}-\rho S
$$

i.e. $S$ defines an infinitesimal conformal transformation of the scalar curvature $S$.

Next, since

$$
\nabla_{\mathscr{T}} \operatorname{grad} \rho=3 \rho^{2} \mathscr{T}
$$

one finds by (11), (12), and (42) that

$$
\mathscr{L}_{\mathscr{T}} g(\mathscr{T} Z)=\frac{2 n-3}{n-1} \rho g(\mathscr{T} Z), \quad Z \in \Xi(M) .
$$

Therefore, and on behalf of (57), it follows that

$$
\nabla \operatorname{grad} \rho=\rho^{2} d p+2 \alpha \otimes \operatorname{grad} \rho .
$$

This shows that $\operatorname{grad} \rho$ is a torse forming vector field [15] [13] [9] with $2 \alpha$ as generating form. 
We assume from now on that $M$ is of even dimension, say $n=2 m$, and we suppose that the following 2-form of rank $2 m$ is globally defined on $M$

$$
\Omega=\sum_{i=1}^{m} \omega^{i} \wedge \omega^{i^{*}}, \quad i^{*}=i+m .
$$

Exterior differentiation of (61) gives in combination with (29) that

$$
d^{-2 \alpha} \Omega=0
$$

which shows that $\Omega$ defines a local conformal symplectic structure with $\alpha$ (resp. $\mathscr{T})$ as covector of Lee (resp. vector of Lee).

Next, by (37) it follows that

$$
\mathscr{L}_{\mathscr{T}} \Omega=\rho \Omega
$$

which means that $\mathscr{T}$ defines an infinitesimal conformal transformation of $\Omega$.

Let now $\mathscr{E}_{\alpha}$ be the vector space such that for every $X_{\alpha} \in \mathscr{E}_{\alpha}$

$$
\alpha\left(X_{\alpha}\right)=\text { Cst. }
$$

Denote by

$$
\mu: T M \rightarrow T^{*} M: Z \rightarrow i_{Z} \Omega
$$

the bundle isomorphism defined by $\Omega$. Setting then $\beta=\mu\left(X_{\alpha}\right)$, one gets by (62)

$$
\mathscr{L}_{X_{x}} \Omega=d^{-2 \alpha} \beta+2 c \Omega
$$

and on behalf of (2) one derives

$$
d^{-2 \alpha}\left(\mathscr{L}_{X_{x}} \Omega\right)
$$

Therefore, we conclude that the Lie derivatives $\mathscr{L}_{X_{\alpha}} \Omega$ are also $d^{-2 \alpha}$-exact. Set now

$$
X_{\beta}=\beta^{\#}=\sum_{i=1}^{m}\left(t_{i} e_{i^{*}}-t_{i^{*}} e_{i}\right)
$$

and operate on $X_{\beta}$ by $\nabla$. By (31) and (33), with $a=0$, one calculates that

$$
\nabla X_{\beta}=X_{\beta} \wedge \mathscr{T}
$$

which shows that $X_{\beta}$ is a Killing vector field. Moreover, one can also verify that $\left[\mathscr{T}, X_{\beta}\right]$, i.e. $X_{\beta}$ commutes with $\mathscr{T}$.

Summarizing, we can formulate the following 
THEOREM 4.1. Let $(M, g)$ be the manifold defined in Section 3. Then, the conformal scalar $\rho$ associated with $\mathscr{T}$ is an isoparametric function and $\mathscr{T}$ defines an infinitesimal conformal transformation of the scalar curvature $S$ on $M$ and of the functions $g(\mathscr{T}, Z)(Z \in \Xi(M))$; that is

$$
\begin{aligned}
\mathscr{L}_{\mathscr{T}} S & =\rho S, \\
\mathscr{L}_{\mathscr{T}} g(\mathscr{T}, Z) & =\frac{2 n-3}{n-1} \rho g(\mathscr{T}, Z) .
\end{aligned}
$$

Besides, if $M$ is of even dimension, it admits a conformal symplectic structure $(\Omega, \alpha)$, having $\alpha=\mathscr{T}^{b}$ as covector of Lee, i.e. $d^{-2 \alpha} \Omega=0$, and $\mathscr{T}$ defines an infinitesimal conformal transformation of $\Omega$, i.e. $\mathscr{L}_{\mathscr{T}} \Omega=\rho \Omega$. If $\mathscr{E}_{\alpha}$ is the vector space such that for $X_{\alpha} \in \mathscr{E}_{\alpha}$, one has $\alpha\left(X_{\alpha}\right)=C$ st., then the Lie derivative $\mathscr{L}_{X_{\alpha}} \Omega$ is $d^{-2 \alpha}$-exact and $X_{\beta}$ is a Killing vector field which commutes with $\mathscr{T}$.

\section{Geometry of the Tangent Bundle}

Let now $T M$ be the tangent bundle having as basis the manifold $M$ introduced in Section 3, which is now in addition assumed to be of dimension $2 m$. In the present section we will study the properties of the lifts to the tangent bundle $T M$ of the tensor fields discussed in the previous sections. Denote by $V\left(V^{i}\right)$ the canonical vector field (or Liouville vector field) [5] and consider $\mathscr{B}^{*}=\left\{\omega^{i}, d V^{i} \mid i=1, \ldots 2 m\right\}$ as a covectorial basis of $T M$. Recalling that the complete lift [16] of the 2-form $\omega^{i} \wedge \omega^{j}$ is defined by

$$
\left(\omega^{i} \wedge \omega^{j}\right)^{C}=d V^{i} \wedge \omega^{j}+\omega^{i} \wedge d V^{j},
$$

one derives by reference to $(61)$ that

$$
\Omega^{C}=\sum_{i=1}^{m}\left(d V^{i} \wedge \omega^{i^{*}}+\omega^{i} \wedge d V^{i^{*}}\right), \quad i^{*}=i+m
$$

we remind that one knows from [16] that $\Omega^{C}$ defines an almost symplectic structure on $T M$. Taking the exterior differential of $\Omega^{C}$, one finds by (29)

$$
d \Omega^{C}=\alpha \wedge \Omega^{C} .
$$

Hence, we observe that in the case under consideration the conformal character of $\Omega$ is conserved bij complete lifting; we emphasize the remarkable aspect of this fact, since in general this property is not conserved. Next, since with respect to the vectorial basis $\mathscr{B}=\left\{e_{i}, \partial / \partial V^{i} \mid i=1, \ldots 2 m\right\}$ the Liouville vector field $V$ is expressed by 


$$
V=\sum_{i=1}^{2 m} V^{i} \frac{\partial}{\partial V^{i}}
$$

one may compute from this that

$$
\mathscr{L}_{V} \Omega^{C}=\Omega^{C}
$$

with reference to [5] this shows that $\Omega^{C}$ is homogeneous of degree 1 . Setting now $\rho=c / f^{2}(c=$ const. $)$, and on behalf of (34), we can write that

$$
\alpha=-\frac{d f}{f} \text {. }
$$

In addition, we put

$$
v=\frac{1}{2} \sum_{i=1}^{2 m}\left(V^{i}\right)^{2}
$$

and consider the function

$$
I=f \vee
$$

If we operate on $I$ by the vertical differential operator $d_{V}$, then we find

$$
d_{V}(I)=f \sum_{i=1}^{2 m} V^{i} \omega^{i}
$$

The basic 1-form

$$
\mu=\sum_{i=1}^{2 m} V^{i} \omega^{i}
$$

is also known [16] as the Liouville form on TM (Alternatively, one can also write that $\mu=V^{b}$ ). By (69) one can now derive that

$$
d\left(d_{V} I\right)=f \sum_{i=1}^{2 m} d V^{i} \wedge \omega^{i}=: \psi
$$

Since the 2 -form $\psi$ is clearly of maximal rank on $T M$, the above equation shows that $\psi$ is an exact (or potential) symplectic form. Since $i_{V} \psi=f \mu$, then by reference to [16], we call $\psi$ the canonical symplectic form on $T M$. Invoking (69), we can check that the Lie derivative of $\psi$ with respect to $V$ is given by

$$
\mathscr{L}_{V} \psi=\psi .
$$


Consequently, $\psi$ is (like $\Omega^{C}$ ) also homogeneous of degree 1 . Besides, operating on $\psi$ by the vertical derivative operator $i_{V}$ and invoking (15), leads to

$$
i_{V}(\psi)=0
$$

On basis of (75) and (76) we conclude that $\psi$ is a Finslerian form [4].

Denote by $\partial_{i}$ the Pfaffian derivative with respect to $\omega^{i}$ and set according to [16]

$$
\partial=\sum_{i=1}^{2 m} V^{i} \partial_{i}
$$

Therefore, by reference to [16], the complete lift $\alpha^{C}$ of $\alpha$ is defined by

$$
\alpha^{C}=\left(\partial t_{i}, t_{i}\right)
$$

Next, setting

$$
\beta=\sum_{i=1}^{2 m} t_{i} d V^{i}
$$

one finds

$$
\alpha^{C}=v \alpha+\beta=d v
$$

in which we have used the notation $v:=L \alpha$ for the image of the 1 -form $\alpha$ under the operator $\boldsymbol{L}$ of Yano and Ishihara (see [16]). Equation (80) shows that the complete lift $\alpha^{C}$ is, like $\alpha$, also an exact form. Consider now on $T M$ the following 2-form of rank $4 m$

$$
\phi=\nu(\alpha \wedge \mu+\psi) .
$$

By exterior differentiation and taking into account (73) and (74), one obtains

$$
d \phi=\left(\frac{\alpha^{C}}{v}-\frac{\alpha}{f}\right) \wedge \phi
$$

From the above it follows that $\phi$ defines on $T M$ a second conformal symplectic structure having the exact form $\alpha^{C} / v-\alpha / f$ as covector of Lee. One also finds that

$$
\mathscr{L}_{V} \phi=2 \phi
$$

which shows that $\phi$ is homogeneous of degree 2. Further, let

$$
\mathscr{T}^{V}=\left(\begin{array}{c}
0 \\
t^{i}
\end{array}\right), \quad \text { and } \quad \mathscr{T}^{C}=\left(\begin{array}{c}
t^{i} \\
\partial t^{i}
\end{array}\right)
$$


be the vertical and the complete lift respectively of the conformal section $\mathscr{T}$. By (83) one may write

$$
\mathscr{T}^{V}=\sum_{i=1}^{2 m} t^{i} \frac{\partial}{\partial V^{i}}
$$

and

$$
\mathscr{T}^{C}=\mathscr{T}+v \mathscr{T}^{v}
$$

By (74) and (83) one finds that

$$
\mathscr{L}_{I} \psi=0, \quad \mathscr{L}_{I^{v}} \psi=0, \quad \mathscr{L}_{I} \cdot \psi=0
$$

which shows that the canonical symplectic form $\psi$ is invariant by $\mathscr{T}, \mathscr{T}^{v}$, and $\mathscr{T}^{C}$.

Summarizing, we can formulate the following

THEOREM 5.1. Let TM be the tangent bundle manifold having as basis the manifold in Section 3 which is now in addition assumed to be of even dimension. Let $V, \mu, \psi$, and $L$, be the canonical vector field, the Liouville form, the canonical form on $T M$, and the operator which assigns to 1-forms on $M$ functions on TM, respectively. Then:

(i) $\quad \psi$ is a Finslerian form which is invariant under the conformal section $\mathscr{T}$ and its vertical and complete lifts $\mathscr{T}^{V}$ and $\mathscr{T}^{C}$, respectively;

(ii) the complete lift $\Omega^{C}$ of the conformal symplectic form $\Omega$ on $M$ is a conformal symplectic form on TM, which is $d^{-\alpha}$-exact and homogeneous of degree 1;

(iii) the complete lift $\alpha^{C}$ of $\alpha$ is also an exact form and the 2-form

$$
\phi=v(\alpha \wedge \mu+\psi)
$$

defines a second conformal symplectic form on TM, having the exact form

$$
\frac{\alpha^{C}}{v}-\frac{\alpha}{f} .
$$

as covector of Lee.

\section{References}

[1] T. P. Branson, Conformally covariant equations and differential forms, Comm. in partial differential equations 7(4) (1982), 393-431.

[2] B. Y. Chen, Geometry of Submanifolds, M. Dekker, New York (1973). 
[3] J. Dieudonné, Treatise on Analysis Vol. 4, Academic Press, New York (1974).

[4] C. Godbillon, Géometrie différentielle et mécanique analytique, Herman, Paris (1969).

[5] F. Guedira, A. Lichnerowicz, Geometrie des algebres de Lie locales de Kirilov, J. Math. Pures Appl. 63 (1984), 407-494.

[6] J. Klein, Espaces variationnels et mécanique, Ann. Inst. Fourier 4 (1962), 1-124.

[7] P. Libermann, C. M. Marle, Géométrie Symplectique, Bases Théorétiques de la Mécanique, U.E.R. Math. du C.N.R.S. 7 (1986).

[8] A. Lichnerowicz, Les relations intégrales d'invariance et leurs applications a la dynamique, Bull. Sci. Math. 70 (1946), 82-95.

[9] K. Matsumoto, I. Mihai, R. Rosca, On gradient almost torse forming vector fields, Math. J. Toyama Univ. 19 (1996), 149-157.

[10] W. A. Poor, Differential Geometric Structures, Mc Graw Hill, New York (1981).

[11] R. Rosca, On parallel conformal connections, Kodai Math. J. 2 (1979), 1-9.

[12] R. Rosca, Exterior concurrent vectorfields on a conformal cosymplectic manifold admitting a Sasakian structure, Libertas Math. (Univ. Arlington, Texas) 6 (1986), 167-174.

[13] C. Udriste, Properties of torse forming vector fields, Tensor NS 42 (1982), 134-144.

[14] K. Yano, On torse forming directions in Riemannian spaces, Proc. Imp. Acad. Tokyo 20 (1944), 340-345.

[15] K. Yano, Integral formulas in Riemannian Geometry, M. Dekker, New-York (1970).

[16] K. Yano, S. Ishihara, Differential Geometry of Tangent and Cotangent Bundles, M. Dekker, New York (1973).

Filip Defever, Department Industriële Wetenschappen en Technologie Katholieke Hogeschool Brugge-Oostende Zeedijk 101, 8400 Oostende, BELGIUM

Radu Rosca, 59 Avenue Emile Zola

75015 Paris, FRANCE 